\title{
Chloride cotransporter NKCC1 inhibitor bumetanide protects against white matter injury in a rodent model of periventricular leukomalacia
}

Lauren L. Jantzie',2, Melody Y. Hu', Hyun-Kyung Park', Michele C. Jackson'1, Jenny Yu', Jessie R. Maxwell² and Frances E. Jensen ${ }^{1,3}$

BACKGROUND: Periventricular leukomalacia (PVL) is a major form of preterm brain injury. $\mathrm{Na}^{+}-\mathrm{K}^{+}-\mathrm{Cl}^{-} 1$ cotransporter (NKCC1) expression on neurons and astrocytes is developmentally regulated and mediates $\mathrm{Cl}^{-}$reversal potential. We hypothesized that NKCC1 is highly expressed on oligodendrocytes (OLs) and increases vulnerability to hypoxia-ischemia (HI) mediated white matter injury, and that the NKCC1 inhibitor bumetanide would be protective in a rodent PVL model.

METHODS: Immunohistochemistry in Long-Evans rats and PLP-EGFP transgenic mice was used to establish cell-specific expression of NKCC1 in the immature rodent brain. HI was induced on postnatal day 6 (P6) in rats and the protective efficacy of bumetanide $(0.3 \mathrm{mg} / \mathrm{kg} / \mathrm{i} . \mathrm{p} . \mathrm{q} 12 \mathrm{~h} \times 60 \mathrm{~h})$ established.

RESULTS: NKCC1 was expressed on OLs and subplate neurons through the first 2 postnatal weeks, peaking in white matter and the subplate between P3-7. Following HI, NKCC1 is expressed on OLs and neurons. Bumetanide treatment significantly attenuates myelin basic protein loss and neuronal degeneration $7 \mathrm{~d}$ post-HI.

CONCLUSION: Presence and relative overexpression of NKCC1 in rodent cerebral cortex coincides with a period of developmental vulnerability to $\mathrm{HI}$ white matter injury in the immature prenatal brain. The protective efficacy of bumetanide in this model of preterm brain injury suggests that $\mathrm{Cl}^{-}$transport is a factor in PVL and that its inhibition may have clinical application in premature human infants.

$\mathbf{P}$ reterm infants make up $\sim 12.5 \%$ of all live births in the United States, and improvements in neonatal clinical care have resulted in an $85-95 \%$ survival rate among those born with very low birth weight $(<1,500 \mathrm{~g})(1)$. However, up to $60 \%$ of these children experience neurological disorders, including cognitive, motor, social, and/or behavioral deficits or delays that may persist throughout the lifespan $(2,3)$. The high incidence of these comorbid disabilities, coupled with the high annual socioeconomic costs of care they are estimated to incur, represent a major public health issue (4).
Preterm infants are particularly susceptible to episodes of hypoxia-ischemia (HI) with or without systemic inflammatory insults both in utero and during the perinatal period. This is due to many factors related to their developmental immaturity including their inadequate lung capacity, frequent variations in blood pressure, underdeveloped cerebral vasculature, and decreased antioxidant reserve (5-7). Cerebral HI can cause a variety of lesions in the developing brain, however the most common manifestation in infants born preterm is periventricular leukomalacia (PVL). PVL is a condition seen in over 50\% of very-low-birth-weight infants $(6,7)$, and is characterized by white matter necrosis and oligodendrocyte (OL) injury, which can present in diffuse and/or cystic forms. Immature OLs and subplate neurons comprise subcortical WM between 24 and 32 wk gestation (8), the developmental stage and window of peak vulnerability to PVL. The most severe cases involve death of all cellular constituents of the white matter, leading to the formation of cellular macroscopic cysts, although this cystic form of PVL is becoming increasingly rare. Presently, the diffuse form of PVL is the most common form observed in preterm babies, involving injury to late-stage OL progenitors, premyelinating OLs, and immature OLs, as well as subplate, cortical, deep gray and cerebellar neurons, culminating in diffuse white and gray matter injury without large areas of focal necrosis $(6,7)$.

The $\mathrm{Na}^{+}-\mathrm{K}^{+}-\mathrm{Cl}^{-}$cotransporter (NKCC1) fluxes sodium, potassium, and chloride ions in to neural cells, contributing to the maintenance of physiological intracellular ion concentrations (9). NKCC1 expression is developmentally regulated in the human and rodent brain, peaking on neurons and astrocytes in early perinatal development before declining in adulthood $(10,11)$. The developmental regulation of NKCC1 on neurons is thought to contribute in part to the increased excitability in the developing brain and to the pathophysiology of neonatal seizures (10,12). Further, in adult animals, NKCC1 is upregulated on neurons and astrocytes following focal ischemia $(13,14)$, with NKCC1 knockout mice exhibiting significantly reduced gray and white matter injury (15), suggesting 
that the cotransporter plays a role in ischemic pathophysiology. However, the role of NKCC1 in HI-induced white matter injury in an established immature animal model has not been investigated, nor has the expression of the transporter on OLs been previously described.

Bumetanide is an inhibitor of NKCC1 and its isoform NKCC2, and is US FDA-approved for clinical use as a diuretic in all patient populations, including neonates. While NKCC2 expression is limited to the kidney, NKCC1 is expressed more widely throughout the brain. Bumetanide effectively crosses the blood-brain barrier (12). Previously, we have shown that NKCC1 facilitates epileptiform discharges in the neonatal rodent brain, and that bumetanide attenuates epileptiform activity and enhances phenobarbital efficacy in a rat model of neonatal hypoxic seizures $(10,12)$. Currently, bumetanide is under study in a phase I/II clinical trial as a single add-on therapy to existing phenobarbital treatment for neonatal seizures in infants 33-44 wks of age at dosages of $0.1-0.3 \mathrm{mg} / \mathrm{kg}$ (clinicaltrials.gov, NCT00830531). Interestingly, autistic behaviors, one of the developmental outcomes associated with PVL in survivors of preterm birth, have been shown to decrease in a small cohort of human subjects following bumetanide treatment $(16,17)$; bumetanide treatment has also been shown to restore abberant behavior and brain oscillations in animal models of autism when given prenatally (18).

In the present study, we evaluated whether NKCC1 was a relevant therapeutic target in immature brain injury, and tested the preclinical efficacy of the NKCC1 inhibitor bumetanide on white matter damage when delivered for $60 \mathrm{~h}$ following $\mathrm{HI}$ in a P6 rat. Bumetanide reduces neuronal and astrocytic injury in adult rodent models of ischemia, with protective mechanisms related to maintenance of intracellular ion gradients, reduced edema, and preservation of $\mathrm{Ca}^{2+}$ homeostasis. Additionally, bumetanide attenuates neonatal seizures in a term-equivalent

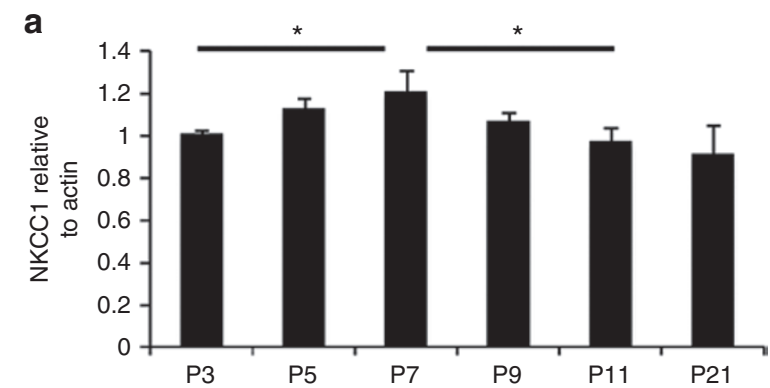

b

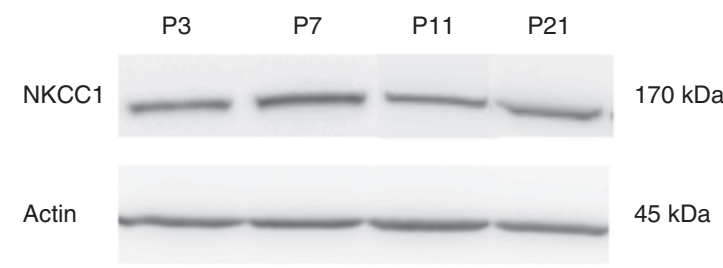

Figure 1. NKCC1 is expressed in developing white matter. Following microdissection of white matter from naive rat pups, Western blot reveals significant NKCC1 expression from P3 through P21. NKCC1 expression is highest at P7 compared to P3 and P11 (a,b). Measurements represent values relative to $\mathrm{P} 3\left(n=6-9 /\right.$ group, $\left.{ }^{*} P<0.05\right)$. rodent model of hypoxia, and can augment the neuroprotective effects of phenobarbital and hypothermia in a rat model of term hypoxic-ischemic encephalopathy $(10,12,19)$. Here, we investigated the relationship between NKCC1 expression and OL and neuronal HI injury in our preterm rodent model of PVL. To unambiguously study NKCC1 expression on OLs, we utilized PLP-EGFP transgenic mice, in which EGFP is expressed in OLs of all developmental stages (20), to complement our studies in immature rats. We hypothesized that NKCC1 is expressed on OLs, subplate, and cortical neurons during the preterm period, coinciding with the peak of white matter vulnerability to PVL, and that administration of bumetanide in a post-HI dosing regimen would protect against white matter injury in our preterm-equivalent rodent model of PVL.

\section{RESULTS}

\section{NKCC1 Expression in the White Matter Peaks Early in the}

\section{Perinatal Period}

The end of the first postnatal week (P5-7) in rodents is a period of heightened white matter vulnerability to HI compared to earlier or later ages $(21,22)$. We have previously shown that this developmental stage is associated with higher levels of NKCC1 in cortical homogenates compared to adult (10). To determine whether NKCC1 is developmentally expressed in white matter in the immature rodent brain, we microdissected white matter from rat pups P3-P21 ( $n=6-9 /$ group) and evaluated expression using immunoblotting (Figure 1). From P3 to P7, there was a $27 \%$ increase NKCC1 protein expression in the white matter (Figure 1a, $P<0.05$ ). Evaluation of the time course of NKCC1 expression after P7 revealed a gradual decline, culminating in a $24 \%$ decrease from P7 to P11 (1.21 \pm 0.09 vs. $0.97 \pm 0.05, P<0.05)$ and $5 \%$ decrease from P11 to P21. Together, these data indicate that NKCC1 is expressed in the white matter during the first 3 postnatal weeks, with a relative upregulation from P3-7, a time of heighted vulnerability to white matter injury.
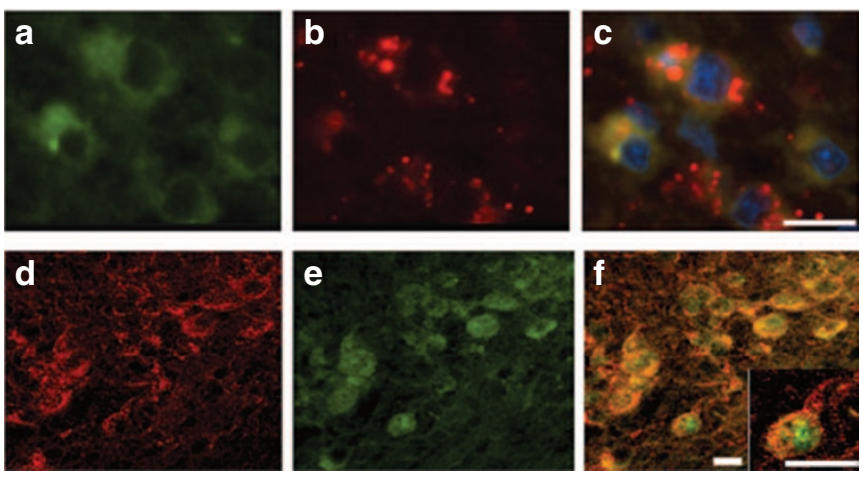

Figure 2. NKCC1 is expressed on immature oligodendrocytes and subplate neurons in the developing rat brain. Fluorescent immunohistochemistry demonstrates NKCC1 (a, green) is highly expressed on GalC-positive immature oligodendrocytes (b, red) in naive rats at postnatal day $6(\mathrm{P} 6, \mathbf{c}$, overlay (yellow with 4',6-diamidino-2-phenylindole (DAPI)-positive nuclei, scale bar $=10 \mu \mathrm{m}))$. Similarly, NKCC1 $(\mathbf{d}$, red) is highly expressed on NeuNpositive subplate neurons (e, green) at the same time point in development (f, overlay, yellow). Scale bars $=20 \mu \mathrm{m}$ outer panel and $10 \mu \mathrm{m}$ inset. 


\section{NKCC1 Is Expressed on Developing OLs and Neurons in the Immature Rodents}

We have shown that white matter and developing OLs are vulnerable to HI insult at P6 in our rat model of PVL $(23,24)$. As previously described, the majority of OLs at P6 in the periventricular white matter and cortex are $\mathrm{O} 4$ and $\mathrm{O} 1$ positive and not myelin basic protein (MBP) expressing $(21,25)$. To test the hypothesis that NKCC1 was present on developing premyelinating OLs in white matter, immunostaining was done in the naive immature rat by double labeling for NKCC1 and the cell marker GalC (Figure 2a-c). Indeed, colocalization of NKCC1 and GalC was observed in white matter from P3 to P14 rats on the cell membranes typical of premyelinating OLs, consistent with this developmental stage ( $n=3-4$ /group). Double labeling with $\mathrm{NKCC1}$ and the neuronal marker NeuN also revealed NKCC1 expression on both cortical and subplate neurons (Figure 2d-f).

To unambiguously identify expression patterns of NKCC1 on OLs, we used immunofluorescence to label NKCC1 at P6 in naive PLP-EGFP transgenic mice ( $n=3-4$ /group), in which EGFP is expressed in OLs of all developmental stages (20). In accordance with our white matter Western blot data and colocalization experments in the rat, NKCC1 was found localized to the cell bodies and/or processes of the majority of EGFP-positive OLs in both cortex and white matter (Figure 3, arrows), confirming baseline NKCC1 expression on OLs during development in vivo. In naive PLP-EGFP pups, we found intense NKCC1 labeling on EGFP-positive OLs exhibiting bipolar or unipolar morphology on both cell bodies and processes, which was relatively increased from P3-P7 compared to later ages (Figure $4, n=3-4$ /group). Similar to immature rats, robust NKCC1 labeling was also observed on the somata and processes of neurons in these developing mice (Figure 3, arrowheads) confirming that this expression pattern is not species-specific. Additionally, a novel finding

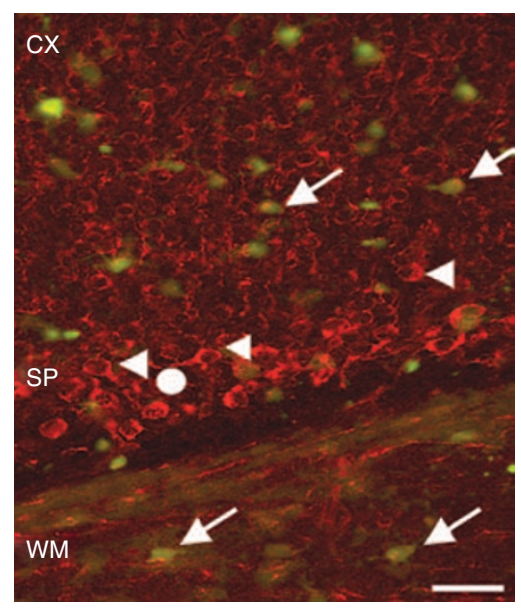

Figure 3. NKCC1 is widely expressed on neurons and oligodendrocytes in naive PLP-EGFP mice. On P6, NKCC1 expression is visible and colocalized (red) to EGFP-positive oligodendrocytes (green) in the white matter and cortex (arrows). NKCC1 expression is also visible in neurons (arrow heads) throughout the cortical mantle and subplate (circle). Scale bar $=20 \mu \mathrm{m}$. in the rodents was that subplate neurons, which were identified by their characteristic morphology and location at the border of cortex and white matter, highly expressed NKCC1 in the first postnatal week (Figure 3, circle). Taken together, these results indicate that NKCC1 is expressed in immature rodent white matter on developing OLs, as well as subplate and cortical neurons, during the peak period of susceptibility to PVL. As increases in NKCC1 expression following injury have been previously reported in epilepsy, stroke, chronic pain, and spinal cord injury $(14,15,26,27)$, we then investigated whether NKCC1 expression on OLs and neurons changes following HI in the immature rodent. As in naive animals, immunostaining for NKCC1 in both P6 Long Evans rats and PLP-EGFP mice revealed $\mathrm{NKCC} 1$ on OLs and neurons in the hemisphere ipsilateral to ligation at 6 and $24 \mathrm{~h}$ post-HI (Figure $5, n=4$ /group).

a
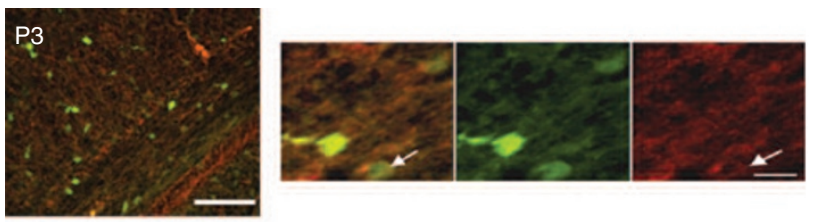

b
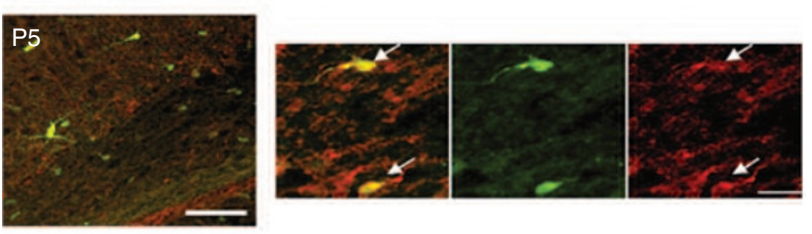

C
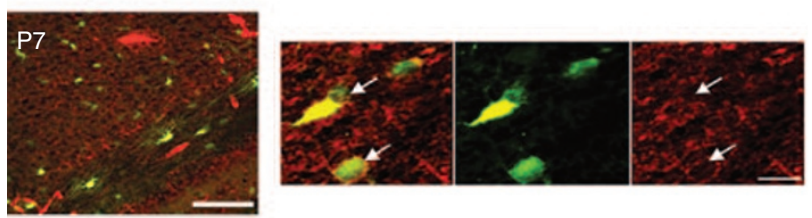

d
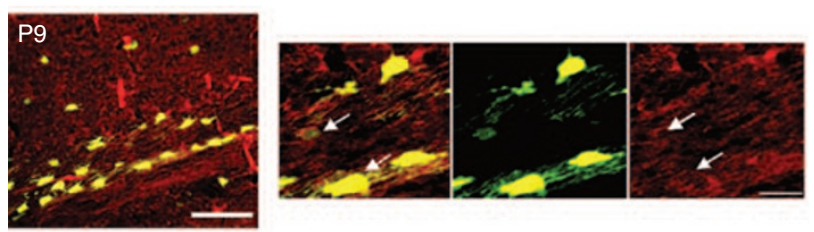

e
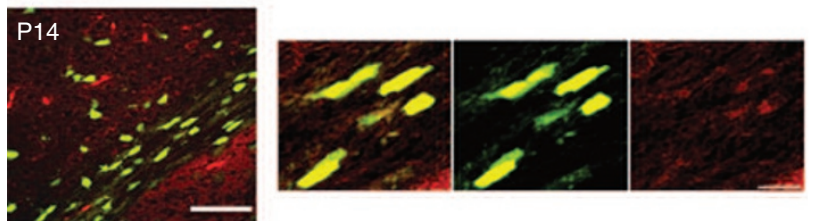

Figure 4. NKCC1 is expressed on developing EGFP-positive oligodendrocytes throughout P3 to P13. In naive PLP-EGFP mouse pups intense NKCC1 labeling (red) on EGFP-positive oligodendrocytes (green) is observed on cell bodies and processes of those exhibiting bipolar or unipolar morphology (a-e). Compared to P14 (e), expression on these oligodendrocytes is relatively increased from P3 to P7 (a-c, arrows). Scale bars $=50$ and $20 \mu \mathrm{m}$ (inset). 

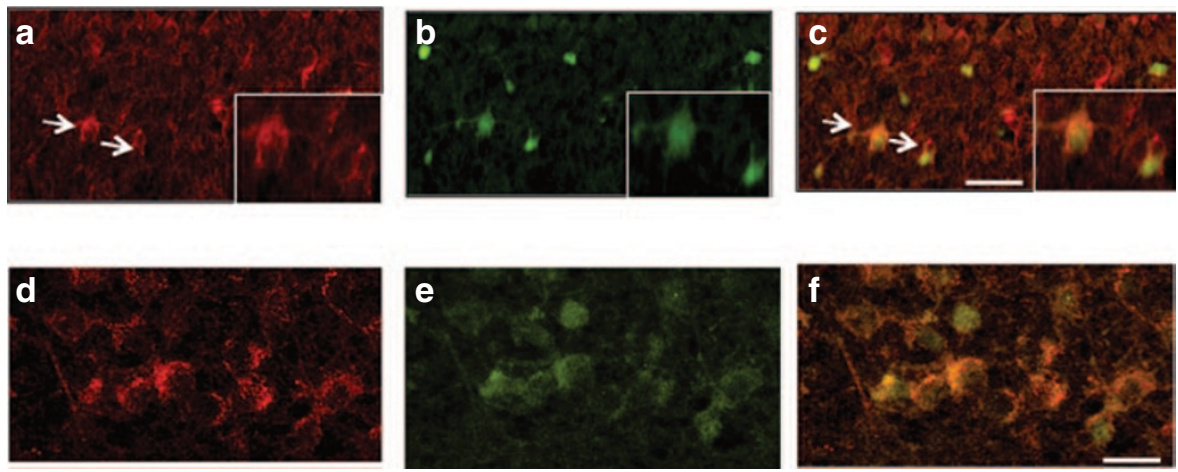

Figure 5. NKCC1 is expressed on oligodendrocytes and neurons following hypoxia-ischemia (HI). Following $\mathrm{HI}$ on postnatal day 6, NKCC1 (a,d, red) is expressed on EGFP-positive oligodendrocytes (b, green) and neurons (e, green) in the cortex at $24 \mathbf{h}$ (c,f). Arrows indicate NKCC1-positive oligodendrocytes. Scale bar $=20 \mu \mathrm{m}$.
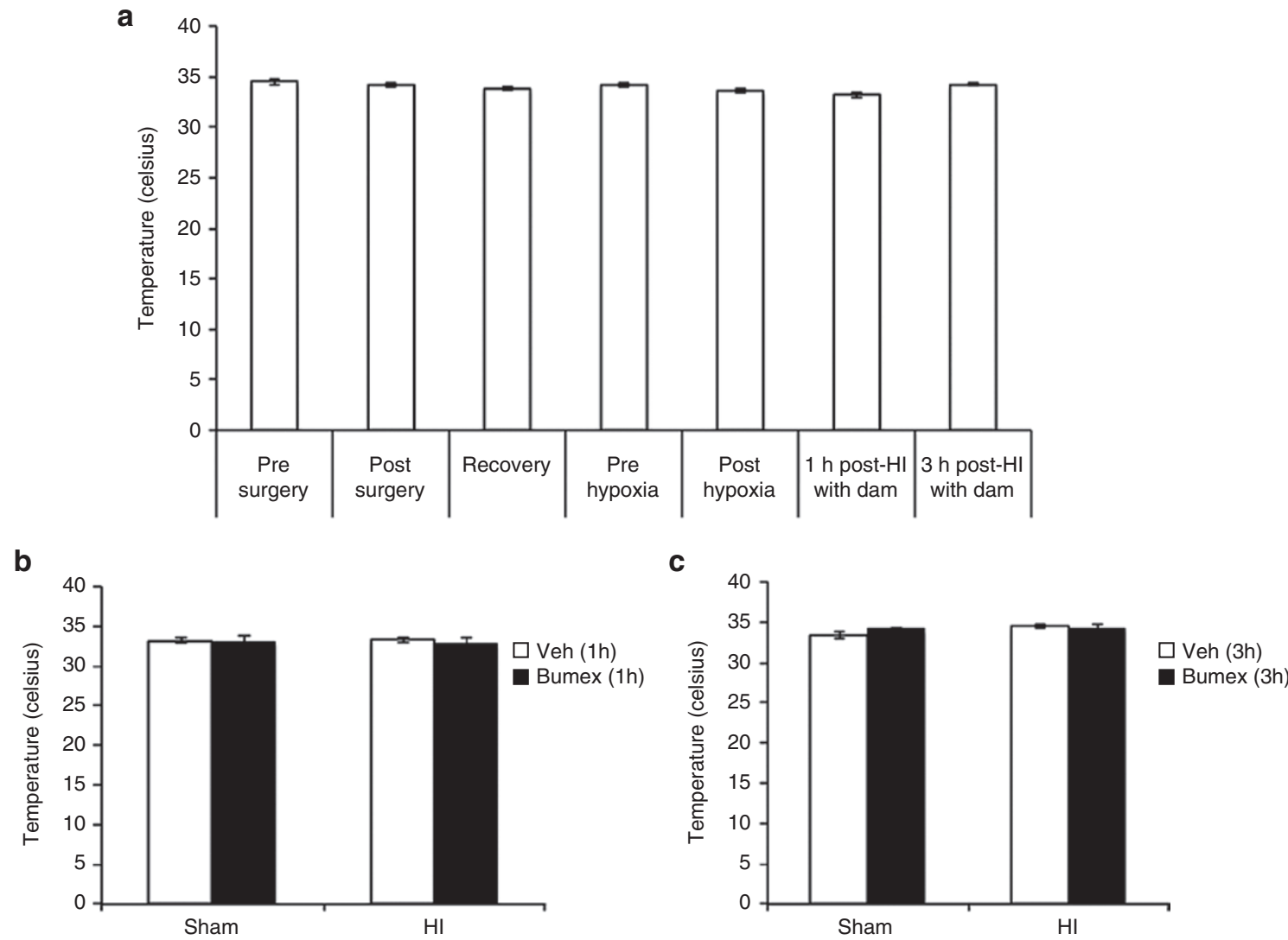

Figure 6. Induction of hypoxia-ischemia and administration of bumetanide does not affect neonatal rat core temperature. Pup core body temperature was recorded before and after carotid ligation, during recovery, before and after hypoxia and in the postoperative period following bumetanide and vehicle administration (a, $n=7-15 /$ group). Bumetanide administration did not alter core temperature compared to vehicle (b,c, $n=7-9 / g r o u p)$. Vehicle treated $=$ white bars, bumetanide treated $=$ black bars.

\section{Bumetanide Attenuates HI-Induced Cerebral Cortical and White Matter Injury}

Given that NKCC1 is present in premature rodent white matter, is developmentally upregulated during a period of heightened susceptibility to $\mathrm{HI}$ and present on OLs following HI, we tested the protective efficacy of the NKCC1 inhibitor bumetanide, administered in a postinsult dosing regimen to Long Evans rats. Rectal temperatures were recorded before and after carotid ligation, during recovery, before and after hypoxia and in the postoperative period following bumetanide and vehicle administration (Figure 6). There were no significant differences in temperature observed at any time point examined (Figure 6a), and the neither the induction of ischemia, nor hypoxia, significantly changed pup core temperature $(34.5 \pm 0.3$ preligation vs. $34.2 \pm 0.2$ postligation; $34.2 \pm 0.2$ prehypoxia vs. $33.7 \pm 0.2$ posthypoxia). Similarly, bumetanide administration did not alter core temperature compared to vehicle (33.6 vs. $33.7^{\circ} \mathrm{C}$; Figure $6 b, c)$. 
a

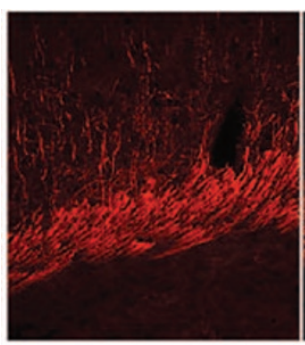

c

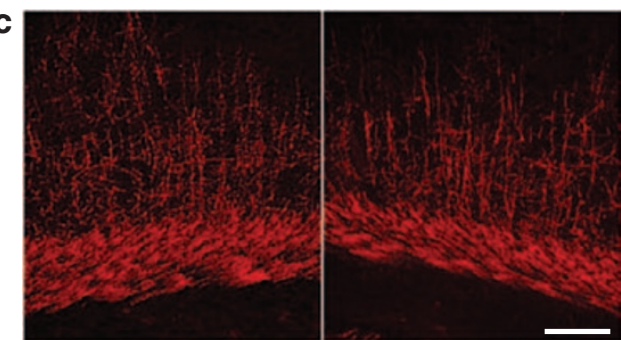

b
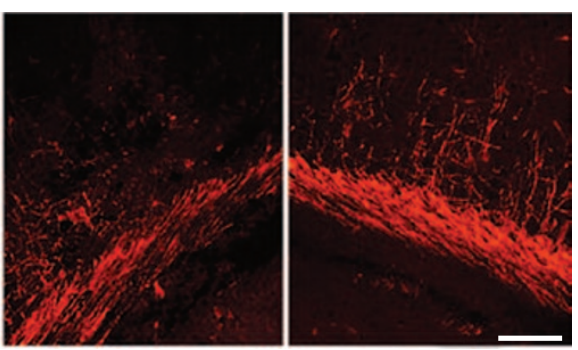

d

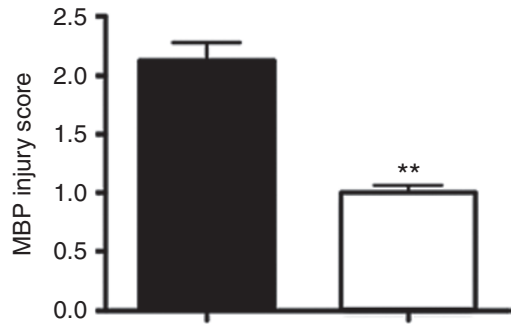

Figure 7. Bumetanide attenuates myelin basic protein loss following hypoxia-ischemia (HI). Compared to sham (a), animals subjected to HI onP6 (b) have significantly reduced MBP in the hemisphere ipsipateral to carotid ligation $7 \mathrm{~d}$ postinjury. MBP protein loss is mitigated by bumetanide treatment $(0.3 \mathrm{mg} / \mathrm{kg} \mathrm{q} 12$ for $60 \mathrm{~h}, \mathbf{c})$ when administered in a postinjury dosing regimen and significantly decreased injury scores. (d, vehicle treated = black bar, bumetanide treated $=$ white bar $).\left(n=7-9 /\right.$ group, $\left.{ }^{* *} p<0.01\right)$. Scale bar $=100 \mu \mathrm{m}$.

We used MBP expression, a marker of mature myelin, at $7 \mathrm{~d}$ following injury as an outcome measure of white matter health and integrity. In vehicle-treated HI rats, MBP expression was significantly reduced in the hemisphere ipsilateral to carotid ligation compared to the contralateral hemisphere and sham controls (Figure 7a,b), similar to our prior reports (24). Treatment with bumetanide $(0.3 \mathrm{mg} / \mathrm{kg}$ q12 for $60 \mathrm{~h}, n=7-9$ / group) immediately following $\mathrm{HI}$ attenuated this loss of MBP observed at P13 (Figure 7c,d), with injury scores were reduced by $56 \%$ in the bumetanide treated pups $(0.8 \pm 0.1)$ compared to those pups receiving vehicle treatment $(2.2 \pm 0.2)$ (Figure $7 d$, $P<0.01)$.

Given the expression of NKCC1 in neuronal subpopulations in cortical gray matter $(10,12)$, we next evaluated whether bumetanide treatment offered any protection in the immature cortex. To assess cortical damage and degeneration, we scored the amount of FJ-positive cells in the cortical mantle overlying the periventricular white matter ( $n=7-9$ /group). In the cortex of vehicle-treated HI pups, mild-to-moderate amounts FJ staining was observed at $7 \mathrm{~d}$ in the hemisphere ipsilateral to carotid ligation, indicative of ongoing cell death. However, treatment with $0.3 \mathrm{mg} / \mathrm{kg}$ bumetanide significantly reduced the number of FJ-positive cells in the cortical mantle overlying the white matter ipsilateral to carotid ligation $7 \mathrm{~d}$ following $\mathrm{HI}$ (Figure 8) including layers 4, 5, and subplate. FJ injury scoring confirmed a significant decrease in degenerating cells between vehicle- and bumetanide-treated pups (Figure 8d), with injury scores reduced by $60 \%$ in the bumetanide-treated pups $(0.5 \pm 0.3)$ compared to those pups receiving vehicle $(1.5 \pm 0.2)$ (Figure 8d, $P<0.01$ ).

\section{DISCUSSION}

In this study, we report for the first time the relative overexpression of NKCC1 on developing OLs in rodent postnatal a

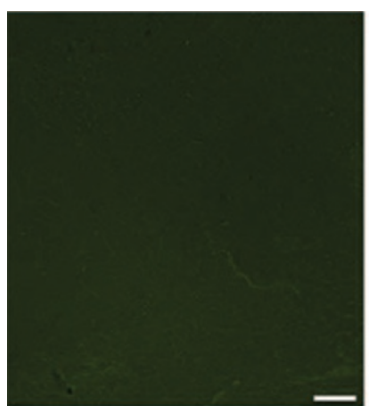

C

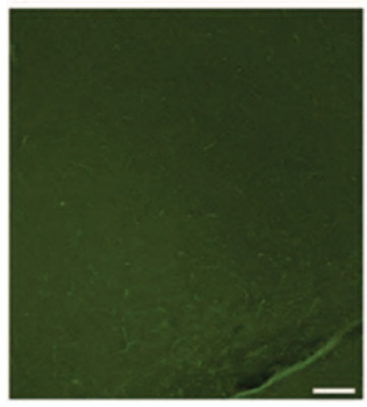

b

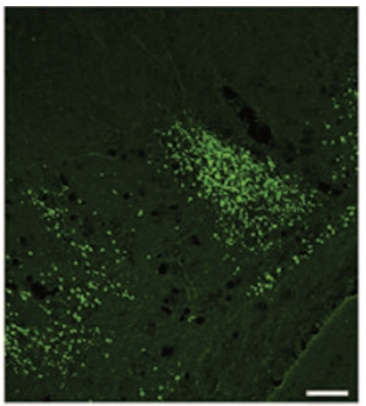

d

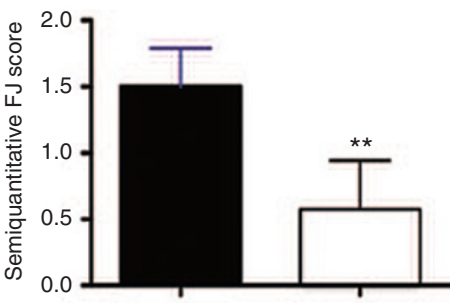

Figure 8. Bumetanide attenuates cellular degeneration following hypoxia-ischemia (HI). Compared to sham (a), animals subjected to $\mathrm{HI}$ on P6 (b) have significantly more fluorojade-positive cells in the hemisphere ipsilateral to carotid ligation $7 \mathrm{~d}$ postinjury. This fluorjade-positive cellular degeneration $7 \mathrm{~d}$ post-HI is mitigated by bumetanide (bumex, $0.3 \mathrm{mg} / \mathrm{kg}$ q12 for $60 \mathrm{~h}, \mathrm{c}$ ) administered in a postinjury dosing regimen. Bumetanide attenuates fluorojade injury scores (d, vehicle treated $=$ black bar, bumetanide treated $=$ white bar. $\left(n=7-9 /\right.$ group, $\left.{ }^{* *} P<0.01\right)$. Scale bar $=100 \mu \mathrm{m}$.

white matter. Consistent with this expression pattern, treatment with the NKCC1 inhibitor bumetanide attenuates white matter and neuronal injury following $\mathrm{HI}$ in preterm equivalent 
neonatal rats. These data provide validation of the expression of this target on vulnerable cell populations in the developing rodent brain and implicate NKCC1 on OLs in HI-mediated injury during a peak period of vulnerability. Taken together, expression of NKCC1 on OLs, subplate, and cortical neurons during the preterm period may confer a unique vulnerability to $\mathrm{HI}$ cell death and the features of PVL, a common diagnosis in preterm infants associated with significant life-long morbidity.

Considerable interest has been directed at $\mathrm{Cl}^{-}$cotransporters and their role in reestablishing hyperpolarizing GABAergic signals in neurons and maintaining ionic homeostasis across cell membranes, including ongoing clinical trials of the NKCC1 inhibitor bumetanide in reducing neonatal seizures. Across species and under normal conditions in neurons, intracellular $\mathrm{Cl}^{-}$is under the control of developmentally regulated cation- $\mathrm{Cl}^{-}$cotransporters that transport $\mathrm{Cl}^{-}$ions with sodium and/or potassium. With advancing age, there is a progressive reduction in intracellular $\mathrm{Cl}^{-}$due to upregulation of $\mathrm{KCC}$, which promotes $\mathrm{Cl}^{-}$efflux and concomitant downregulation of NKCC1 (11). While KCC2 is primarily a neuronal transporter, NKCC1 has been reported on glia in the adult central nervous

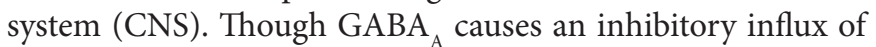
$\mathrm{Cl}^{-}$in the adult brain, $\mathrm{GABA}_{\mathrm{A}} \mathrm{R}$ binding in the immature brain can be differentially excitatory or inhibitory depending on the intracellular $\mathrm{Cl}^{-}$gradient and the shifting of polarity, which is likely mediated in part by the developmental expression of NKCC1. Indeed, preclinical studies indicate that following stroke, in epilepsies, and spinal cord injuries, levels of $[\mathrm{Cl}]_{\mathrm{i}}$ are elevated leading to changes in neuronal ion flux, excitatory GABA action, hyperexcitability, and cell death. Despite this, in vivo expression of NKCC1 in the cellular components of developing white matter has not previously been reported and the putative role of this transporter in the pathophysiology of $\mathrm{HI}$ in the premature brain following injury has not been explored.

Here, we show that post-treatment with bumetanide, a NKCC1 inhibitor, is protective in a rodent model of premature brain injury at when administered at $0.3 \mathrm{mg} / \mathrm{kg}$ q12 for $60 \mathrm{~h}$. This dosage is comparable to the upper end of the dosages being investigated in human newborns with refractory seizures $(0.1-0.3 \mathrm{mg} / \mathrm{kg}$ dose escalation, clinical trials.gov NCT00830531). Bumetanide has an established safety profile as a diuretic in the pediatric patient population and has been used since 1986 in children to treat hypertension, bronchopulmonary dysplasia, nephritic syndrome, and congestive heart failure (28). It has a relatively short half-life and crosses the blood-brain barrier in neonatal rodents (29). There are several potential mechanisms by which bumetanide may be beneficial in $\mathrm{OL}$ and neuronal injury, including preservation GABAergic signaling and of ionic homeostasis across cell membranes, prevention of osmotic overload and interaction with $\mathrm{Na}^{+}$/ $\mathrm{Ca}^{2+}$ exchangers, and modulation of alpha-amino-3-hydroxy5-methyl-4-isoxazole propionic acid receptor (AMPAR) and $\mathrm{N}$-methyl-d-aspartate receptor (NMDAR). NKCC1 is present and active on cultured OLs, and prior preclinical data suggests it maintains intracellular $\mathrm{Cl}^{-}$concentrations above electrochemical equilibrium and is required for GABAergic trophic effects, normal GABAR activation, and signal transduction (30). In addition, bumetanide may protect OLs by promoting ionic homeostasis and preventing osmotic overload, cytotoxic edema, and massive calcium influx. Specifically, NKCC1's role as an $\mathrm{Na}^{+}$-importer, in conjunction with the activity of $\mathrm{Na}^{+} / \mathrm{Ca}^{2+}$ exchanger proteins, has been shown in animals to cause pathological $\mathrm{Ca}^{2+}$ increases in OLs, cortical neurons, and astrocytes $(31,32)$. NKCC1 has also been linked to AMPAR and NMDAR function and activity. In cultured OLs, AMPAR activation induces NKCC1 phosphorylation increasing its activity and maintaining high $\left[\mathrm{Cl}^{-}\right]_{\mathrm{i}}, \mathrm{Na}^{+}$influx, and mitochondrial $\mathrm{Ca}^{2+}$ accumulation leading to release of cytochrome $\mathrm{c}$ and apoptosis. In this context, AMPAR activation induced cell death and was attenuated by NKCC1 blockade (31). This phenomenon has also been reported on neurons, strengthening a possible link between NKCC1 and glutamatergic excitotoxicity (33), and may be a developmentally regulated mechanism given elevated AMPAR, NMDAR, and NKCC1 expression on immature OLs and neurons in both the preterm rodent and human brain (34). NKCC1 expression may also indirectly facilitate NMDAR activity on both neurons and OLs by increasing intracellular $\mathrm{Cl}^{-}$concentrations, which can repel NMDA channel-blocking $\mathrm{Mg}^{2+}$ ions, allowing for excessive $\mathrm{Ca}^{2+}$ influx and another mechanism for excitotoxic cell death (35).

In addition to the protective effect on white matter, our data also confirm bumetanide's ability to protect neurons from degeneration following HI injury in the developing rodent brain. Developing neocortical neurons are potentially vulnerable to the direct and indirect effects of depolarizing GABA receptors due to developmental regulation of NKCC1 as well as glutamate receptors, as previously discussed. In termequivalent rats, bumetanide has been shown to augment the neuroprotective efficacy of phenobarbital plus hypothermia following HI- and hypoxia-induced seizures without causing apoptosis $(12,19)$. Taken together with our findings showing protection of white and gray matter following ischemic injury in preterm equivalent rats, these data indicate that $\mathrm{NKCC1}$ is a modifiable molecular target in the developing brain.

Children born before $32 \mathrm{wks}$ gestation are at high risk for epilepsy, cognitive delay, behavioral problems, and cerebral palsy. Currently, the only treatments available to these children are limited to symptom relief and of limited efficacy. Together with the data presented here, recent advances suggest that cation $\mathrm{Cl}^{-}$ cotransporters, including NKCC1 and KCC2, may contribute to the pathophysiology of injury in the developing brains of these infants (36). Human tissue studies have shown the elevation of NKCC1 in cerebral cortex of the premature brain (10), and samples from infants with confirmed white matter damage show dysregulated expression of both NKCC1 and KCC2 in subplate and white matter (36). Neuroprotective agents such as bumetanide administered during the neonatal period to infants at high risk for brain injury and chronic deficits may attenuate abnormalities in $\mathrm{Cl}^{-}$cotransporter expression and function, optimize the balance of excitation and inhibition in the developing brain, and facilitate synaptogenesis and circuit formation, thereby decreasing the chronic neurological impairments in these children. Any 
potential risks of chronic bumetanide therapy are likely to be substantially outweighed by its putative benefit if brief treatment confers protection for both white and gray matter injury in the setting of preterm hypoxic-ischemic brain injury. Together, the data presented here suggest therapeutic efficacy and clinical utility of $\mathrm{Cl}^{-}$cotransporter-directed therapies and support further consideration and study of brief bumetanide therapy in human premature brain injury. Future investigations in our laboratories will address the limitations of the current study including the putative sex effects or different responses to bumetanide treatment in male and female pups throughout the lifespan, and potential impact on functional outcomes in long-term survival animals. Examination of potential sex differences in transporter function throughout development and following injury with emphasis on cell death mechanisms, behavioral assessment following treatment, and mechanistic studies focused on the link between NKCC1, GABA receptor subunits and KCC2 chloride cotransporters would also be beneficial.

\section{METHODS \\ Animals \\ Male mouse and rat pups were used in this investigation to establish baseline NKCC1 expression on OLs and putative bumetanide effi- cacy in HI-induced white matter injury. Both Long-Evans rat pups (Charles Rivers Laboratories, Wilmington, MA) and proteolipid protein-enhanced green fluorescent protein (PLP-EGFP) transgenic mouse pups (B6/CBA background) $(20,37)$ were maintained in a tem- perature-controlled animal care facility with a 12-h light-dark cycle. All procedures were approved by and in accordance with the guide- lines of the Animal Care and Use Committees at Children's Hospital (Boston, MA) and the University of New Mexico Health Sciences Center, and the US National Institutes of Health's Guide for the Care and Use of Laboratory Animals.}

\section{HI}

At P6, which corresponds to $31-36$ of gestation $(25,38,39)$ pups underwent unilateral carotid artery ligation followed by hypoxia $(6 \%$ $\mathrm{O}_{2}$ balanced $\mathrm{N}_{2}$ for $1 \mathrm{~h}$ ) as described previously (24). In brief, animals were anesthetized with ether $(24,40)$, with each surgery completed with in $7 \mathrm{~min}$. The proximal internal carotid artery was then isolated and permanently ligated with 4-0 silk. Sham animals had their carotid artery isolated but not ligated and were not exposed to hypoxia. Naive animals were subject neither to hypoxia nor sham surgery, and were not exposed to anesthesia. The incision in the neck was then closed and sealed with dermabond, and the pups allowed to recover for $1 \mathrm{~h}$ on a thermal circulating water blanket to maintain core temperature at $33.5-34.5^{\circ} \mathrm{C}$. The animals were then placed on a thermal, circulating water blanket, in sealed hypoxia chamber infused with $6 \% \mathrm{O}_{2}$ balanced nitrogen for $1 \mathrm{~h}$. Following recovery from hypoxia, the pups were returned to their dam until euthanasia. To unambiguously evaluate and confirm NKCC1 expression on OLs following $\mathrm{HI}$ and the expression levels seen on developing rat OLs, PLP-EGFP mouse pups $(n=4$ /group) underwent unilateral carotid artery ligation followed by hypoxia $\left(7 \% \mathrm{O}_{2}\right.$ balanced $\mathrm{N}_{2}$ for $\left.40 \mathrm{~min}\right)$. In mice, this paradigm of hypoxia following ischemia yields a similar pattern and distribution of periventricular and subcortical white injury and gray matter damage, including MBP loss and Fluoro-Jade positive degenerating cells, as observed in our Long Evans rat model.

Naive, sham and HI rodents were euthanized along an extended time course from $6 \mathrm{~h}$ to $7 \mathrm{~d}$ post-HI. Briefly, animals were deeply anesthetized with sodium pentobarbital and transcardially perfused. Brains were extracted, postfixed overnight in $4 \%$ paraformeldahyde, and then cryoprotected in 30\% sucrose. For Western blot analyses, rats were decapitated and brains rapidly removed. Brains were placed on a precooled dissecting block and white matter rapidly dissected via micropunch.

\section{Bumetanide Administration}

Rats in the bumetanide treatment group received intraperitoneal injections of bumetanide $(0.3 \mathrm{mg} / \mathrm{kg}$ dissolved in $15 \%$ dimethyl sulfoxide (DMSO, Sigma Aldrich, St Louis, MO) balanced sterile saline) beginning immediately following hypoxia and continuing every $12 \mathrm{~h}$ for $60 \mathrm{~h}$, per brain and serum levels experimentally determined in our laboratory $(12,29)$. Pups received a total of six doses. Animals in the vehicle-treated groups were injected with $15 \%$ DMSO in sterile saline ( $n=7-9 /$ group). Injection volumes did not exceed $100 \mu$ l. All pups were monitored throughout the course of experimental treatment for health, including vitals such as weight gain, hydration, and body temperature. Each rodent in this study, regardless of outcome measure and experimental endpoint was monitored for fluctuations in core body temperature over the course of experimentation. The rectal temperature of each pup was recorded using a thermocouple thermometer designed for neonatal rodents (Physitemp, Clifton, NJ) prior to ischemia, immediately following ischemia, during recovery, prior to hypoxia, immediately posthypoxia, 1 and $3 \mathrm{~h}$ following hypoxia and bumetanide administration (Figure 1). No significant differences were found in these metrics between any of the treatment groups, and no animals required subcutaneous fluids at any time as determined by skin turgor via tenting, milk band presence, pup coloring and skin appearance, suckling, and weight gain.

\section{Western Blot Analysis}

Microdissected white matter tissue from three pups were pooled and homogenized (total $n=6-9 /$ group), sonicated, centrifuged, and membrane proteins quantified using a Bradford protein assay (BioRad, Berkeley, CA). Thirty micrograms of membrane protein were then equalized, and loaded on a $4-20 \%$ Tris-glycine gel. After electrophoresis, proteins were transferred to polyvinylidene difluoride membranes, blocked, and incubated with anti-NKCC1 (Aviva Systems Biology, San Diego, CA, at 1:500), or actin antibodies (Sigma at $1: 5,000)$ overnight. The following day, membranes were probed with species appropriate anti-rabbit or anti-mouse HRP conjugated secondary antibodies. Blots were imaged on a GE LAS 4000 Image reader and relative optical density for each band was quantified using GE ImageQuant Software and standardized to actin to correct for equal protein loading (Piscataway, NJ).

\section{Histological Analysis and Immunofluorescence}

Serial 20 - $\mu \mathrm{m}$ coronal brain sections were cut on a Leica cryostat from the anterior extent of the lateral ventricles through the posterior extent of the dorsal hippocampus and collected on slides (Superfrost Plus, Fisherbrand, Waltham, MA). Sections were rinsed and blocked for $1 \mathrm{~h}$ in a $10 \%$ normal goat serum solution. Primary antibodies against myelin basic protein (anti-MBP, Covance, Princeton, NJ, 1:500), or immature OLs (anti-galactocerebroside C (GalC), Millipore, Bellerica, MA, 1:100) or neurons (anti-neuronal nuclei (NeuN), Millipore, 1:500) were then applied and sections were allowed to incubate overnight at $4{ }^{\circ} \mathrm{C}$. The following day, sections were washed, and a species-appropriate fluorescent secondary antibody conjugated to Alexa-Fluor Red 568 (1:1,000, Invitrogen, Carlsbad, CA) or AlexaFluor Green 488 (1:500, Invitrogen) was applied for $1 \mathrm{~h}$. Sections undergoing double labeling were again washed, blocked, and in some cases incubated with antibodies against NKCC1 (anti-NKCC1, Aviva Systems Biology,1:500) overnight at $4{ }^{\circ} \mathrm{C}$. Subsequently, an antirabbit secondary antibody conjugated to either Alexa-Fluor Red 568 (1:1,000, Invitrogen) or Alexa-Fluor Green 488 (1:500, Invitrogen) was applied for $1 \mathrm{~h}$. Slides were then washed and coverslipped with Fluoromount aqueous mounting medium (Sigma-Aldrich).

\section{Assessment of Myelination and MBP Scoring}

White matter injury severity in animals $7 \mathrm{~d}$ post-HI (P13) was assessed semiquantitatively as per our previously published methods (24). In brief, MBP staining in the periventricular WM was compared ipsilateral and contralateral to the ligation ( $n=7-9$ /group). The regions of interest for this study were the medial and lateral fields of the periventricular white matter at the level of the middorsal hippocampus, $2.8-3.1 \mathrm{~mm}$ from bregma and $2.6-3.0 \mathrm{~mm}$ lateral to midline. Images were taken in three sections per brain, resulting in a total of six comparison points per hemisphere per animal. MBP loss/white 
matter injury severity was quantified by a blinded observer on a scale of $0-5$. Animals $7 \mathrm{~d}$ post-HI, exhibited nearly fully developed myelin, and the criteria in comparing ipsilateral injury to contralateral were as follows: 0 = no difference in MBP staining; 1 = disorganization and/or mild loss of cortical processes; 2 = moderate loss of cortical processes; $3=$ complete loss of cortical processes with intact capsule; $4=$ loss of cortical processes with thinning and/or breaking capsule; $5=$ complete loss of processes with near-complete to complete loss of capsule. A mean score was obtained for the regions of interest, and the lesion severity was compared between the vehicle- and bumetanide-treated groups.

\section{Fluorjade-B Staining and Assessment of Cell Death}

To label degenerating cells with Fluorojade-B, sections were dehydrated in a series of ethanol washes and then incubated in a $0.06 \%$ potassium permanganate solution for $10 \mathrm{~min}$ to suppress background. Sections were then washed with $\mathrm{ddH}_{2} 0$ and incubated in a $0.001 \%$ FluoroJade solution made in $0.1 \%$ acetic acid for $20 \mathrm{~min}$. After water wash, the sections were placed in a drying oven held at $37.5^{\circ} \mathrm{C}$ until completely dry. Sections were placed in xylene $(1 \mathrm{~min})$ and coverslipped with DPX ((Distrene, Plasticiser, Xylene) Sigma Aldrich).

Gray matter injury $7 \mathrm{~d}$ post-HI (P13) was assessed as per our previously published methods ( $n=7-9 /$ group) (26). In brief, the extent of FluoroJade staining was compared in hemispheres ipsilateral and contralateral to the ligation in two sections per brain. Injury severity was quantified on a scale of $0-3$, as follows: $0=$ no FJ-positive cells; $1=$ diffuse areas of mild FJ staining; 2 = moderate staining occurring in dense, focal or columnar patches; 3 = widespread, severe staining distributed through the entire brain region. A mean score was obtained for each animal, and the injury severity was compared between the vehicle- and bumetanide-treated groups.

\section{Statistical Analyses}

Data are expressed as mean \pm SEM. Normally distributed data differences between two groups were compared using the Student's twotailed $t$-test. For multiple comparisons among normally distributed data in three or more groups, a one-way ANOVA with Tukey's post $h o c$ analysis was used. A $P$ value of $<0.05$ was considered significant.

\section{ACKNOWLEDGMENTS}

The authors are particularly appreciative of Shenandoah Robinson for helpful discussions regarding the manuscript.

\section{STATEMENT OF FINANCIAL SUPPORT}

This work was supported by the US National Institute of Neurological Disorders and Stroke at the National Institutes of Health (NS031718 and DP1 OD003347 from the Office of the Director to F.E.J.), the Heart and Stroke Foundation of Canada (to L.L.J.), the William Randolph Hearst Foundation at Harvard Medical School (to L.L.J.), the University of New Mexico Department of Pediatrics (to L.L.J.), and Alberta Innovates Health Solutions Canada (to L.L.J.).

Disclosures: The authors report they have nothing to disclose.

\section{REFERENCES}

1. Martin JA; Centers for Disease Control and Prevention (CDC). Preterm births - United States, 2007. MMWR Surveill Summ 2011;60 Suppl:78-9.

2. Fetters L, Huang HH. Motor development and sleep, play, and feeding positions in very-low-birthweight infants with and without white matter disease. Dev Med Child Neurol 2007;49:807-13.

3. Gurses C, Gross DW, Andermann F, et al. Periventricular leukomalacia and epilepsy: incidence and seizure pattern. Neurology 1999;52:341-5.

4. Behrman RE, Butler AS. Preterm Birth: Causes, Consequences, and Prevention. Washington, DC: The National Academies Press, 2007.

5. Ferriero DM. Oxidant mechanisms in neonatal hypoxia-ischemia. Dev Neurosci 2001;23:198-202.

6. Volpe JJ. The encephalopathy of prematurity-brain injury and impaired brain development inextricably intertwined. Semin Pediatr Neurol 2009;16:167-78.

7. Volpe JJ. Brain injury in premature infants: a complex amalgam of destructive and developmental disturbances. Lancet Neurol 2009;8:110-24.

8. Back SA, Luo NL, Borenstein NS, Levine JM, Volpe JJ, Kinney HC. Late oligodendrocyte progenitors coincide with the developmental window of vulnerability for human perinatal white matter injury. J Neurosci 2001;21:1302-12.

9. Russell JM. Sodium-potassium-chloride cotransport. Physiol Rev 2000;80:211-76.

10. Dzhala VI, Talos DM, Sdrulla DA, et al. NKCC1 transporter facilitates seizures in the developing brain. Nat Med 2005;11:1205-13.

11. Kaila K, Price TJ, Payne JA, Puskarjov M, Voipio J. Cation-chloride cotransporters in neuronal development, plasticity and disease. Nat Rev Neurosci 2014;15:637-54

12. Cleary RT, Sun H, Huynh T, et al. Bumetanide enhances phenobarbital efficacy in a rat model of hypoxic neonatal seizures. PLoS ONE 2013;8:e57148.

13. Lenart B, Kintner DB, Shull GE, Sun D. Na-K-Cl cotransporter-mediated intracellular $\mathrm{Na}+$ accumulation affects $\mathrm{Ca} 2+$ signaling in astrocytes in an in vitro ischemic model. J Neurosci 2004;24:9585-97.

14. Yan Y, Dempsey RJ, Flemmer A, Forbush B, Sun D. Inhibition of $\mathrm{Na}(+)$ $\mathrm{K}(+)-\mathrm{Cl}(-)$ cotransporter during focal cerebral ischemia decreases edema and neuronal damage. Brain Res 2003;961:22-31.

15. Chen H, Luo J, Kintner DB, Shull GE, Sun D. Na(+)-dependent chloride transporter (NKCC1)-null mice exhibit less gray and white matter damage after focal cerebral ischemia. J Cereb Blood Flow Metab 2005;25: $54-66$.

16. Lemonnier E, Ben-Ari Y. The diuretic bumetanide decreases autistic behaviour in five infants treated during 3 months with no side effects. Acta Paediatr 2010;99:1885-8.

17. Lemonnier E, Degrez C, Phelep M, et al. A randomised controlled trial of bumetanide in the treatment of autism in children. Transl Psychiatry 2012;2:e202.

18. Tyzio R, Nardou R, Ferrari DC, et al. Oxytocin-mediated GABA inhibition during delivery attenuates autism pathogenesis in rodent offspring. Science 2014;343:675-9.

19. Liu Y, Shangguan Y, Barks JD, Silverstein FS. Bumetanide augments the neuroprotective efficacy of phenobarbital plus hypothermia in a neonatal hypoxia-ischemia model. Pediatr Res 2012;71:559-65.

20. Mallon BS, Shick HE, Kidd GJ, Macklin WB. Proteolipid promoter activity distinguishes two populations of NG2-positive cells throughout neonatal cortical development. J Neurosci 2002;22:876-85.

21. Dean JM, Moravec MD, Grafe M, et al. Strain-specific differences in perinatal rodent oligodendrocyte lineage progression and its correlation with human. Dev Neurosci 2011;33:251-60.

22. Jensen FE. Developmental factors regulating susceptibility to perinatal brain injury and seizures. Curr Opin Pediatr 2006;18:628-33.

23. Follett PL, Deng W, Dai W, et al. Glutamate receptor-mediated oligodendrocyte toxicity in periventricular leukomalacia: a protective role for topiramate. J Neurosci 2004;24:4412-20.

24. Jantzie LL, Talos DM, Selip DB, et al. Developmental regulation of group I metabotropic glutamate receptors in the premature brain and their protective role in a rodent model of periventricular leukomalacia. Neuron Glia Biol 2010;6:277-88.

25. Talos DM, Fishman RE, Park H, et al. Developmental regulation of alphaamino-3-hydroxy-5-methyl-4-isoxazole-propionic acid receptor subunit expression in forebrain and relationship to regional susceptibility to hypoxic/ischemic injury. I. Rodent cerebral white matter and cortex. J Comp Neurol 2006;497:42-60.

26. Hasbargen T, Ahmed MM, Miranpuri G, et al. Role of NKCC1 and KCC2 in the development of chronic neuropathic pain following spinal cord injury. Ann N Y Acad Sci 2010;1198:168-72.

27. Kahle KT, Staley KJ, Nahed BV, et al. Roles of the cation-chloride cotransporters in neurological disease. Nat Clin Pract Neurol 2008;4:490-503.

28. Sullivan JE, Witte MK, Yamashita TS, Myers CM, Blumer JL. Pharmacokinetics of bumetanide in critically ill infants. Clin Pharmacol Ther 1996;60:405-13

29. Li Y, Cleary R, Kellogg M, Soul JS, Berry GT, Jensen FE. Sensitive isotope dilution liquid chromatography/tandem mass spectrometry method for quantitative analysis of bumetanide in serum and brain tissue. J Chromatogr B Analyt Technol Biomed Life Sci 2011;879:998-1002.

30. Wang H, Yan Y, Kintner DB, Lytle C, Sun D. GABA-mediated trophic effect on oligodendrocytes requires $\mathrm{Na}-\mathrm{K}-2 \mathrm{Cl}$ cotransport activity. J Neurophysiol 2003;90:1257-65. 
31. Chen H, Kintner DB, Jones M, et al. AMPA-mediated excitotoxicity in oligodendrocytes: role for $\mathrm{Na}(+)-\mathrm{K}(+)-\mathrm{Cl}(-)$ co-transport and reversal of $\mathrm{Na}(+) / \mathrm{Ca}(2+)$ exchanger. J Neurochem 2007;102:1783-95.

32. Luo J, Wang $\mathrm{Y}$, Chen $\mathrm{H}$, et al. A concerted role of $\mathrm{Na}+-\mathrm{K}+-\mathrm{Cl}-$ cotransporter and $\mathrm{Na}+/ \mathrm{Ca} 2+$ exchanger in ischemic damage. J Cereb Blood Flow Metab 2008;28:737-46.

33. Beck J, Lenart B, Kintner DB, Sun D. Na-K-Cl cotransporter contributes to glutamate-mediated excitotoxicity. J Neurosci 2003;23:5061-8.

34. Jantzie LL, Talos DM, Jackson MC, et al. Developmental Expression of N-Methyl-d-Aspartate (NMDA) Receptor Subunits in Human White and Gray Matter: Potential Mechanism of Increased Vulnerability in the Immature Brain. Cereb Cortex 2015;25:482-95.

35. Ben-Ari Y, Khazipov R, Leinekugel X, Caillard O, Gaiarsa JL. GABAA, NMDA and AMPA receptors: a developmentally regulated 'ménage à trois'. Trends Neurosci 1997;20:523-9.
36. Robinson S, Mikolaenko I, Thompson I, Cohen ML, Goyal M. Loss of cation-chloride cotransporter expression in preterm infants with white matter lesions: implications for the pathogenesis of epilepsy. J Neuropathol Exp Neurol 2010;69:565-72.

37. Fuss B, Mallon B, Phan T, et al. Purification and analysis of in vivo-differentiated oligodendrocytes expressing the green fluorescent protein. Dev Biol 2000;218:259-74.

38. Clancy B, Darlington RB, Finlay BL. Translating developmental time across mammalian species. Neuroscience 2001;105:7-17.

39. Dean JM, Bennet L, Back SA, McClendon E, Riddle A, Gunn AJ. What brakes the preterm brain? An arresting story. Pediatr Res 2014;75: 227-33.

40. Ma JY, Zhang SP, Guo LB, et al. KCC2 expression changes in Diazepamtreated neonatal rats with hypoxia-ischaemia brain damage. Brain Res 2014;1563:22-30. 